\title{
Managing Intellectual Property Rights in the WWW: Patterns and Semantics
}

\author{
Rosa Gil \\ Dpt. de Tecnología UPF \\ P. de Circumval·lació 8 \\ 08003 - Barcelona, \\ rosa.gil@upf.edu
}

\author{
Rubén Tous \\ Dpt. de Tecnologia UPF P. \\ de Circumval-lació 8 \\ 08003 - Barcelona \\ ruben.tous@upf.edu
}

\author{
Jaime Delgado \\ Dpt. de Tecnologia UPF P. \\ de Circumval lació 8 \\ 08003 - Barcelona \\ jaime.delgado@upf.edu
}

\begin{abstract}
An architecture to manage Digital Rights, in this case Intellectual Property Rights IPR, in the World Wide Web is being developed. It has as main features: semantics and complex systems analysis. Semantics allow establishing concepts meaning inside the architecture while statistics allow managing a huge amount of semantic information. In order to provide an efficient performance, some aspects have been enriched as digital rights information representation and database management. Semantic graphs encapsulate digital rights information and statistical analysis over the stored graphs in the semantic database allows extracting behavioral patterns. It is possible to distinguish four relevant phases: Retrieval IPR from the World Wide Web performed by web crawlers, IPR semantic representation, IPR statistical analysis in the semantic database and the last part concludes with action answers over DRM as negotiation aspects.
\end{abstract}

\section{Introduction}

Digital Rights Management is a key issue to achieve in a network system, in our case, we are going to focus in Intellectual Property Rights (IPR). Usually it is know as Copyright. The paper is structured as it follows.

In section 2, the title summarizes the content: 'Searching IPR in the World Wide Web' is not an automatic process, different possibilities are found about this topic; after an exhaustive survey, we propose a method for gathering copyright information over the World Wide Web.

Following this part, we find the semantic contribution. Section 3 explains Semantic Web [1] languages and why they have been chosen. They are used to represent data coming from the WWW survey done in section 2. This information is stored semantically in a database. The data is structured as a graph; the advantages for doing it are explained in this section as well as the suitable languages. These languages come from Semantic Web. For instance, as a root for IPR representation IPROnto[2], a legal ontology designed by us, is used as well as its connections to MPEG-21 rights expression language representations in XML [3].

Following language description, we understand the reasons why the representation language is relevant to apply the statistical analysis. Thus, a new research line, which is focused in how a network behaves, is developed. In this case our network is a knowledge network as we are going to see. The network is represented by a graph, thus statistical analysis is applied to a graph that posses a huge amount of nodes (for us, it means concepts). The graph is based on a semantic language for knowledge representation and constitutes a knowledge network. Usually a network with a great number of nodes is difficult to manage; this is the reason why statistical analysis is applied. The result of applying gives patterns as 'clustering' and phenomenon as 'small-world' or 'scale-free' properties. In this section it is demonstrated that it is possible to extract some patterns over Semantic Web. An exhaustive statistical analysis has been made over Semantic Web and pieces of it, i.e. legal ontologies.

The last part explains the double purpose of the architecture to be designed: Reporting inappropriate DRM uses and negotiating IPR Management.

\section{Searching IPR in the Web}

\subsection{The World Wide Web and IPR}

Because it is worldwide, the original works accessible in the WWW are authored and used by 
authors and users from different legal frameworks. Strictly, we cannot talk about an 'International Copyright' law, which will automatically protect an author's writings throughout the world. Protection against unauthorized use in a particular country depends on the national laws of that country. However, most countries offer protection to foreign works under certain conditions, which have been greatly simplified by international copyright treaties and conventions.

There are two principal international copyright conventions, the Berne Union for the Protection of Literary and Artistic Property (Berne Convention) and the Universal Copyright Convention (UCC). Moreover, there are initiatives to promote a general legal framework in e-commerce as the World Intellectual Property Organization (WIPO) that is going to be used in section 3 to conceptualize Copyright concept. Generally, the works of an author who is a national or domiciliary of a country that is a member of these treaties, or works first published in a member country or published within 30 days of first publication in a Berne Union country may claim protection under them. There are no formal requirements in the Berne Convention. Under the UCC, any formality in a national law may be satisfied by the use of a notice of copyright in the form and position specified in the UCC.

A UCC notice should consist of the copyright symbol ( $\mathrm{C}$ in a circle) accompanied by the year of first publication and the name of the copyright holder.

This notice must be placed in such manner and location as to give reasonable notice of the claim to copyright. However, since the Berne Convention prohibits formal requirements that affect the "exercise and enjoyment" of the copyright, some countries (like the United States) make the use of a copyright notice optional (however the use of a copyright notice still provides certain advantages in some countries, like defeating a defense of "innocent infringement" in the United States).

\subsection{Identifying copyright statements on a web site by software}

A web site can be composed of a lot of different elements, such as hypertext, still or moving images, sound files, programs, and other kind of multimedia content.

Each different element is independent in terms of intellectual property rights, but by default all are copyright protected according to the Berne Convention.
Despite of this, most sites include at least one visible UCC notice covering all the site elements, with the aim to limit 'innocent infringement' or to satisfy laws from countries where it is mandatory.

The UCC notice can appear in combination with more detailed generic or national-specific information about the legal issues concerning the use of the web site and its related elements. This 'passive copyright statements', that can be expressed in natural language or in a machine-understandable format, can be accompanied by some mechanisms to make them effective, or at least difficult to infringe. We call these mechanisms 'active copyright statements', and include e.g. the mouse right-button techniques to difficult undesired downloads.

In order to identify IP information from web sites, it is necessary to determine recognition patterns for the different evidences. The patterns will be used by a web crawler (see section 5), that will traverse all web pages from a specific crawling domain (in our case the '.es' domain) gathering information about subjects, objects and conditions related to copyright information.

\subsection{Passive copyright statements}

Passive copyright statements can come in two different flavors, in natural language (NL) or in some machine understandable format. When expressed in natural language the statements can be written in English or in other language. We have identified some English keywords denoting the presence of an UCC notice or usually present in link labels pointing to web pages with intellectual property information (or about subjects involved with it):

1. All Rights Reserved

2. Read Me

3. Who are We

4. Copyright and Privacy

5. Contact Us

6. Site Info

7. About Us

8. Terms of Use

When expressed in other languages, the evidences of intellectual property sentences use to be direct translations of the same English keywords. Because we are going to target Spanish web pages, we have identified some labels broadly used behind intellectual property information (or about subjects involved with it) in Spain:

1. 'Aviso Legal'

2. 'Contactar'

3. 'Quien somos'

4. 'Condiciones de uso' 
Determining by software the specific conditions related to a NL-expressed passive copyright statement is a difficult issue. There is not a mandatory formal way to express such conditions as can be deduced from the following three examples:

1. From 'FreeFoto.com' (http://freefoto.com/ index.asp): 'FreeFoto.com' is one of the largest collections of free photographs for non-commercial use on the Internet. The comprehensive, yet easy to navigate site features 40 main sections with over 600 sub headings [...] The photographs are free to private users and for sale to commercial users."

2. From Debbie Orlean's Frog Photographs http://www.darsbydesign.com/orlean/frogthumb.htm: "You may use any of my graphics on your site. Here, or any you find browsing my links. Please give me credit and provide a link back here. A simple text link will do. [...] Watermark will be removed from any photos that you order.'

3. From the United States National Library of Medicine (http://www.nlm.nih.gov/about/imagespage. $\mathrm{html}$ ): "Most information at this site is in the public domain. Unless otherwise stated, these documents may be freely distributed and used for non-commercial, scientific, educational or personal purposes. However, you may encounter documents or portions of documents contributed by private companies or organizations."

These three arbitrary examples illustrate how difficult can be to identify specific intellectual property rights or conditions. So in these cases, we just can ambition to identify the subjects and objects of the rights. The second kind of passive copyright statements are those expressed using some machine understandable format. Such metadata, usually based on XML or RDF [4], can take also different forms. Maybe the most used recently is Creative Commons [5], but other metadata schemas allow specifying with more or less detail information related to IPR. While Dublin Core [6] allows us to easily extract some information about the authoring of digital items, ODRL [7] or MPEG-21 [8] offer very detailed metadata concerning all aspects of intellectual property as we are going to see in section 3 .

To process this machine-understandable metadata we will need more than a simple text-based crawler. Because we want to traverse the metadata structure we will need to post-process the documents with metadata information with specific filters, one for each metadata format.

\subsection{Active copyright statements related to still images}

Still images on the web are maybe the worst victims of copyright 'innocent infringement'. Their accessibility and their potential use with or without modifications as part of other works (other web sites or digital/physical design works) motivate a lot of illegal uses.

Because there is no way to prevent anyone from using a screen capture utility (or even the Print Screen key on the keyboard) to capture an image, and because as soon as anyone visits a page, all the images on it are cached (e.g. in Temporary Internet Files when using Windows and Internet Explorer), there is no definite way to protect images from being copied. The only thing we can do is making it more difficult. This can be done using techniques to disable right clicking, encrypt the code, and remove toolbar features. JavaScript is used for most all of these methods, that are dependent on the browser (most of them just work for Internet Explorer), and won't work with less common implementations.

Our target is to identify patterns that allow us to detect these different techniques in a web site, being an evidence of implicit intellectual property manifestation. Following we enumerate some of the most used mechanisms.

\subsubsection{Disabling Right Clicking with JavaScript}

This is the most common technique. When the user tries to right click an image, a message box pops up informing that the action has been disabled. There are many different scripts for disabling right clicking, and they can be programmatically detected by searching some common function calls like:

document.captureEvents(Event.MOUSEDOWN);

\subsubsection{Disabling Right Clicking without JavaScript}

There is another non-JavaScript method to prevent right click saving of images. It consists on putting a transparent gif covering the image (used as a background for a table cell, see Table 1).

Table 1. HTML code for right clicking in order to save a transparent gif

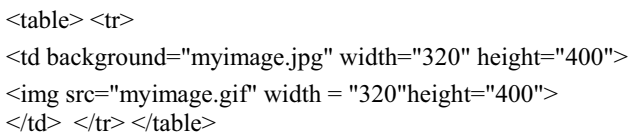




\subsubsection{Disabling image toolbar}

The Image Toolbar feature of IE6 allows to copy images without using right-clicking. It's easy to turn off this feature by inserting this meta tag between the head HML tags:

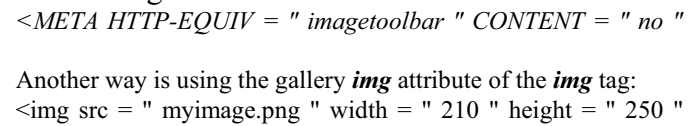

Another way is using the gallery $\boldsymbol{i m g}$ attribute of the $\boldsymbol{i m g}$ tag:

$<$ img src $="$ myimage.png " width $=" 210 "$ height $=" 250 "$ GALLERYIMG $=$ " no " >

\subsubsection{Encrypting the code}

There are many encrypting utilities available on the net like HtmlEncrypt (http :// www. topshareware. com/HTML-Encrypt-download-12472.htm) that allow to encrypt an HTML file.

\subsubsection{Removing toolbars from Internet Explorer with JavaScript}

Another extended method is to operate over navigators. There are many methods to manipulate navigators toolbars. Publicity web sites use them often. JavaScript is the most extended language in manipulating HTML code but it is not the only of course. In this part, as an example, we explain one of these methods that consist on removing completely the toolbar from the Internet Explorer navigator window where the image is being displayed to difficult user actions to download the image sources. This can be achieved by using some parameters of the JavaScript open function:

Table 2. JavaScript open function to difficult user actions to download images

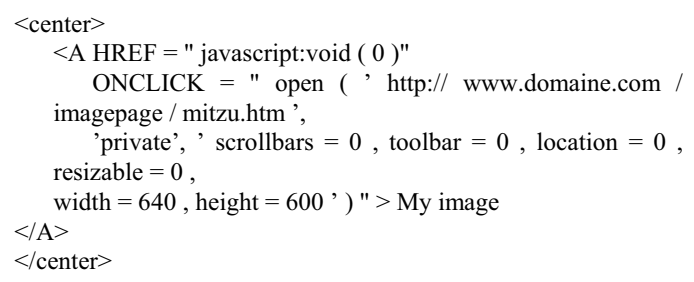

\subsection{Crawling implementation}

We use the open-source Heritrix (http://crawler. archive.org) Java web crawler to traverse the web pages of the 'es' domain. The target is to identify all the evidences of intellectual property expressions and store them in an RDF database. We have developed different Java postprocessors for each specific kind of copyright statement. The post-processors are connected to the crawler, and called when a new web page is processed. Some of them are:

1. For natural language passive copyright statements we use patterns expressed with regular expressions. We just store subjects and objects involved on the copyright.

2. For each kind of metadata passive copyright statement we are implementing a specific postprocessor (Creative-commons, Dublin Core, ODRL and MPEG-21 [3]). In these cases we store all the information in RDF.

3. For the different active copyright statements we use HTML Tidy [9] to convert the code to XHTML and then XQuery [10] to express the different patterns.

We store the subjects and objects involved and also the kind of protection mechanism.

\section{IPR semantic representation}

This section outcomes the rights expression language relevance to achieve an efficient system performance. The rights expression language representation will allow a statistical approach in the next section 4

\subsection{Semantics}

Several markup languages are designed to represent concepts. For instance, web pages are based on HTML and their evolutions as DHTML. This was a revolution that takes its greatest maximum in the web, however something relevant was missing yet.

We refer to semantics, because the amount of information that can be found in the web claimed to have a new way for representing data.

\subsubsection{Languages for expressing rights}

XML (eXtensive Markup Language) is the most widespread metadata representation in new technologies.

However, RDF (Resource Description Language) represents an evolution that uses XML syntax but it is not constrained by that, so RDF is a foundation for processing metadata and it provides interoperability between applications that exchange machineunderstandable information on the Web. RDF emphasizes facilities to enable automated processing of Web resources.

It is a model for describing directed labelled pseudo-graphs. The stucture is as follows, there is a primitive basic Triple: (rdf:Statement) and it is 
composed by: Subject (resource) (rdf:Resource), Predicate (resource) (rdf:Property) and an Object (resource or literal). A Resource is something that can be represented by URI (Universal Resource Identifier).

XML and RDF are defined by schemas, XMLSchema and RDFSchema respectively, which define vocabulary and relationships constraints over the XML tree and the RDF graph.

OWL (Ontology Web language) [11] allows providing more detailed constrains over the RDF graph. An ontology is a specification of a conceptualization. In this context, it is a synonym of "RDF vocabulary", which is basically a collection of classes of nodes and classes of properties. For instance, we can specify elements cardinality, as how many days we need. Classify elements by their properties, i.e. define implicit classes.

These arguments carried us to design and build IPROnto [2] using a language based on graphs. When a call for proposals for Part 5 and Part 6 of MPEG-21 was open, we began to design it using the following supports: the IEEE SUO upper ontology [12] which provided interoperability to other ontologies initiatives, for the specific part of e-commerce <indecs> framework as the core IPR e-commerce specific part [13] was chosen, referring to the legal aspects, we were astonished because of the heterogeneity in the countries laws as we have seen in section 2 , however we found WIPO recommendations [14] which aim to achieve an agreement. In Figure 1, we show only the graph part where copyright is detailed.

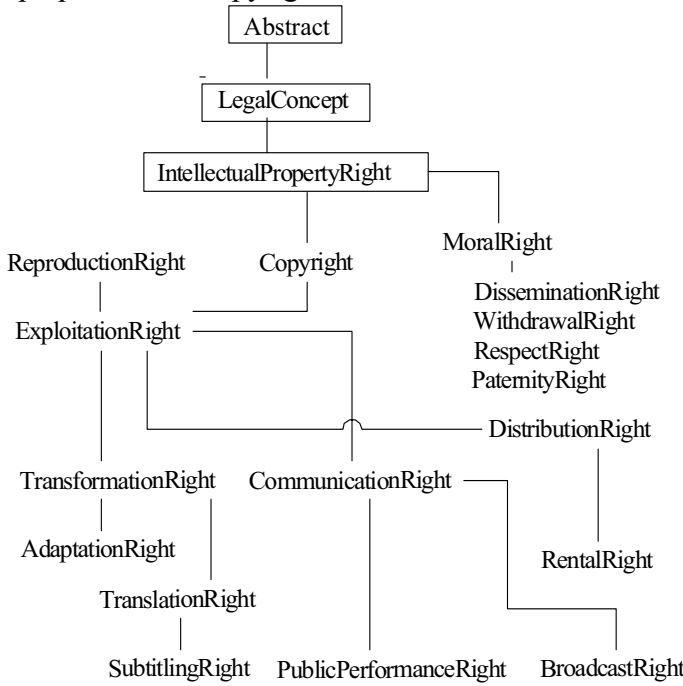

Figure 1. Copyright detailed classification inside IPROnto ontology

This structure has been used to associate the IPR information taken from section 2. However, it has not to be viewed as a way of doing taxonomy with the gathered information in the World Wide Web, the ontology also describes the processes that carry the contract and licenses formalizations in IPR domain. Our group has not forgotten other IPR initiatives, e.g. MPEG-21 that has been also integrated in the semantic framework $[15,16,17]$.

\subsubsection{Describing IPR processes}

Following these lines we find an example of IPROnto concept called IPRTransfer, a generic framework for rights transference between legal parties that hold rights, which are called RightsHolders (one of the IPROnto roles). In this context persons consent the distribution process, the involved RightsHolders or their representatives. There is a Transfer rights (IPRTransfer) in a context which is defined in terms of time and space when there are transferred rights: DistributionRight, ReproductionRight and CommunicationRight. Content Provider is the granter while Media Distributor and RightsHolder is/are (they could not be the same person) the grantee.

The Creation is the object where the transferred rights apply. Sometimes Compensation is given and it represents the counterpart that the rights granter receives for the granted rights.

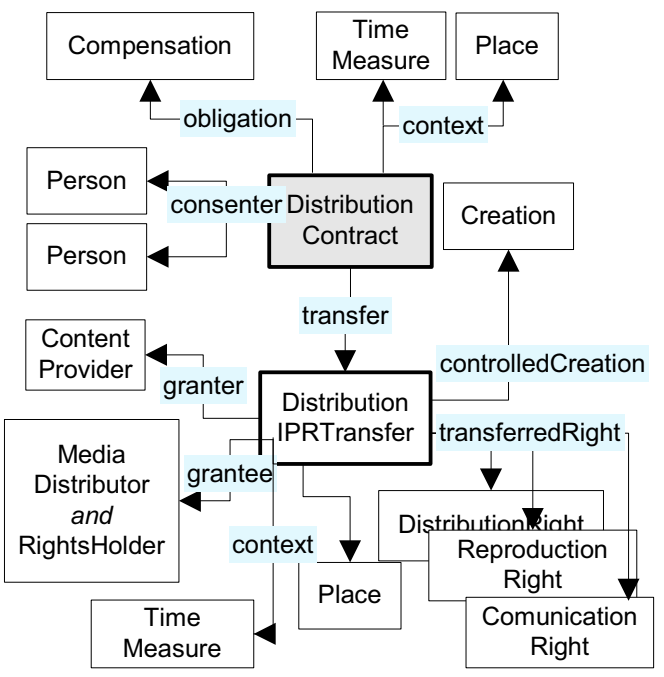

Figure 2. Example of Transferring IPR in IPROnto.

There are other international initiatives that are expressing their licenses in RDF, for instance Creative Commons (http://creativecommons.org/) which offers a flexible range of protections and freedoms for authors and artists. They have built upon the "all rights 
reserved" of traditional copyright to create a voluntary "some rights reserved" copyright. They are a nonprofit organization and all of their tools are free. We are also working to relate these licenses to this format.

\section{IPR statistical analysis}

We found a problem when we were working with too many graphs, since the set of graphs have many nodes to explore when the graphs were store in the database. It was necessary to develop something which allowed us to manage a great amount of information. We know that statistics manages systems that posses an enormous quantity of particles. We thought that this methodology would help us to achieve our goal: finding some stationary graph behavior.

We found the key issue in Complex Systems (CSs) which are made up of the combination of a great amount of elements. However, their behavior is not the sum of the behavior of their parts. Examples of CSs are metabolic networks [18], acquaintance networks [19], food webs [20] or neural networks [21]. Currently, it has also been shown that the WWW is a CS [22]. Graphs are used to model CSs in order to analyze them. Nodes represent the CS parts (chemical components, people, species, neurons, web pages). Edges model the relationships among the parts (chemical reactions, acquaintanceship, species dependences, neuron axons, web links). The resulting graphs show statistical properties that characterize CSs. They are considered sufficient conditions for identifying a CS:

- Degree distribution: the resulting graphs, although they model systems that are shaped without a central control, are not random graphs, as it was first believed. The probability $\mathrm{P}(\mathrm{k})$ that a vertex has a degree $\mathrm{k}$ does not follow a Poisson distribution as in random graphs. Instead, it shows a power-law distribution, $\mathrm{P}(\mathrm{k})=\mathrm{k}^{-\mathrm{r}}(1)$

The $r$ exponent characterizes this kind of distributions. Networks showing this distribution are called scale-free networks [23]. In other words, they show the same properties independently of the scale at which they are observed.

- Small world: a graph is a small world if the average minimum path length $\mathrm{d}$ between vertices is short $[24,25]$, usually scaling logarithmically with the total number of vertices. Graphs showing an average path length similar to random graphs of the same size and average degree are very likely small worlds [26], d $\sim \mathrm{d}_{\text {random }}$

- Clustering coefficient: It measures the probability that two neighbors of a given node are also neighbors of one another. For random graphs it is a small quantity. However, CSs show a high clustering compared to random graphs, $\mathrm{C}>>\mathrm{C}_{\text {random. }}$ A high clustering confirms small-world phenomenon:

\subsection{Semantic Web Graph}

Due to self-similarity and scale invariance of CSs and to show the feasibility of our approach, we can perform this statistical analysis selecting a significant portion of the SemanticWeb and the results can be inferred to other scales. We have focused on the ontological part of the Semantic Web, i.e. we model the graph from a set of semantic web ontologies. We could also use instance metadata but we consider that at this first stage, to focus on ontologies makes the conclusions more relevant. Instance metadata usually models "real networks" that should be analyzed on their own or have already been shown to be CSs. For instance, FOAF metadata [27] models social networks that have been extensively studied as CSs. Therefore, in order to collect the semantic web ontologies that are analyzed, a RDF crawler is launched over a set of Web Ontologies. The processed URIs are combined in a RDF graph built from 1,365,286 triples for 282 ontologies at the DAMLOntology Library [28].

In order to analyze the obtained Semantic Web graph we use Pajek [29], a large networks analysis tool. The RDF triples are translated to Pajek network format. The triples subjects and objects became network nodes connected by directed edges from subject to object. For this first analysis we will focus on the explicit nature of the Semantic Web. Only triples explicitly stated in the processed Ontologies are considered. Therefore, for the moment, the potential triples that could be inferred applying RDF or OWL semantics are ignored. The Pajek network has 307,231 nodes and 588,890 arcs.

Once loaded in Pajek, the available tools are used to obtain the required information about the graph: Average degree and degree distribution and clustering factor. Then multiply the resulting mean value by two in order to compute it for an undirected graph. The Average minimum path length: average over a random selection of 20 nodes, while Power-law tails exponent is a linear regression from the degree distribution.

Table 3. Some CS statistical properties. Networks name, number of nodes, average degree $<\mathrm{k}>$, clustering factor $\mathrm{C}$, average path length $<\mathrm{d}>$ and power-law exponents $\mathrm{r}$

\begin{tabular}{|l|l|l|l|l|l|}
\hline Network & Nodes & $<\mathrm{k}>$ & $\mathrm{C}$ & $<\mathrm{d}>$ & $\mathrm{r}$ \\
\hline
\end{tabular}




\begin{tabular}{|l|l|l|l|l|c|}
\hline $\begin{array}{l}\text { DAMLOntos } \\
(11-04-2003)\end{array}$ & 56,592 & 4.63 & 0.152 & 4.37 & -1.48 \\
\hline $\begin{array}{l}\text { DAMLOntos } \\
(31-01-2005)\end{array}$ & 307,231 & 3.83 & 0.092 & 5.07 & -1.18 \\
\hline WWW & $200 \mathrm{M}$ & 0.108 & 3.10 & -2.24 & - \\
\hline WordNet & 66,025 & 0.060 & 7.40 & -2.35 & - \\
\hline WordsNetwork & 500,000 & 0.687 & 2.63 & -1.50 & - \\
\hline
\end{tabular}

The results of the graph analysis are shown in Table 3. DAMLOntos represent ontologies at DAML library. It can be compared with the same parameters for other CSs networks: the results from some WWW studies [30], WordNet [31] and human language words networks [32].

- From the previous data, we can deduce that the Semantic Web is a small world comparing its average path length $\mathrm{d}=4.37$ and 5.07 to the corresponding value for a random graph with the same size and average degree $<\mathrm{d}>=7.23$.

- Moreover, the clustering factor $\mathrm{C}=0.152$ and 0.092 is much greater than $C_{\text {rand }}=0.0000895$ for the corresponding random graph.

- The final evidence is the degree distribution; it is clearly a power-law. The linear regression of this function gives an exponent $\mathrm{r}=-1.186$ with a regression error $\mathrm{e} \%=0.896$ and the same with $\mathrm{r}=-1.48$ with less error.

The analysis has been repeated for smaller graphs yielding the same conclusion. For instance, for a 971 nodes graph corresponding to the IPROnto ontology: C $=0.071$ while $\mathrm{C}_{\text {rand }}=0.0034272,<\mathrm{d}>=3.99$ while $\mathrm{d}_{\text {rand }}=5.38$ and $\mathrm{r}=-1.06$ with $\mathrm{e} \%=4.45$.

We plan to apply inferences to the retrieved triples in order to check the resulting graph.

\section{Architecture outline}

Before deciding what actions to execute, an overview of the architecture design is presented.

\subsection{Architecture design}

Summarizing the previous sections we provide an architecture design (see Figure 3) where at the bottom part we find database related components as IPR Semantic database and the Statistical Semantic Graph explained in the previous sections. Web crawler is the agent which searches WWW to find IPR content according to active and passive copyright statements as it is explained in section 2 (it is illustrated as little balls connected to the agent). B-Agent manages the actions to be executed depending on its mission. We propose two in the next sections: reporting inappropriate DRM use and negotiating IPR Management.

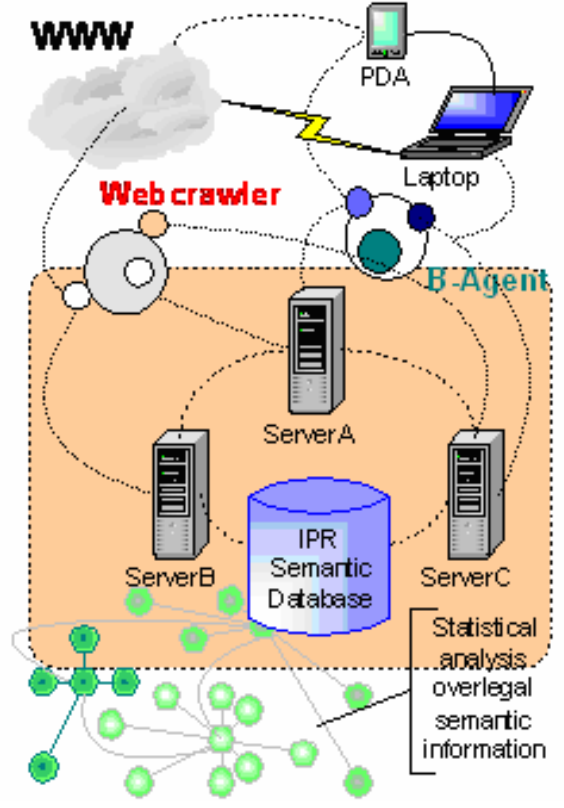

Figure 3. Architecture design

\section{Architecture applications}

We briefly describe some actions that can be done with this architecture relating to IPR domain.

\subsection{Reporting inappropriate DRM}

We have found multimedia content as images, pictures, songs, videos, etc. that have been taken from other web sites and they are exhibited without any copyright reference to the original content.

When a rights holder wants to control how his/her multimedia content rights are managed in the WWW the architecture provides him/her a way to detect inappropriate uses. It is true that it is not an automatic method, however the statistics over the graph are fast to compute, and the information given by the graph is visual. For example, human beings as well as artificial agents can detect nodes that behave as hubs or they are isolated. In the worst case, this methodology gives locations where the probability is higher than trial and error.

\subsection{Negotiating IPR Management}


Another case can be the negotiation of the multimedia material that has been found in the World Wide Web. Agents perform a role as RightsHolder, User, Licensing Agents and so on and negotiate using the adequate IPR protocol. Figure 4 shows the negotiation protocol and the roles.

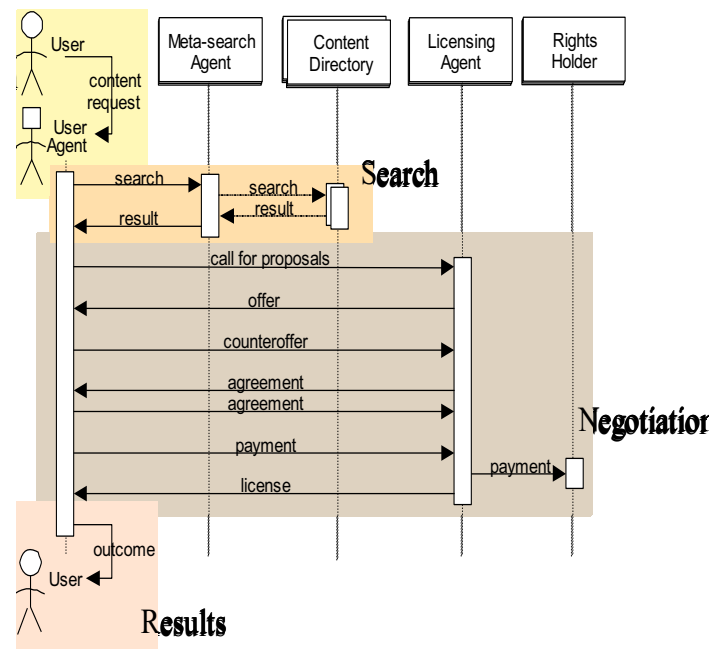

Figure 4. Negotiation protocol

\section{Conclusions and future work}

We have designed a scalable architecture that presents aspects that are not usual to find in this kind of architectures: semantics and patterns due to complex systems properties. This architecture allows us to perform different kind of actions in the IPR domain effectively. We have discussed IPR information in the WWW and how to integrate it in the architecture.

We are considering extending patterns capability to recognize watermarks. Also, we want to extend the analysis to web pages in other languages and compare data analysis.

Finally, we plan to implement specific applications such as tool to find illegal use of copyrighted content in the WWW.

\section{References}

[1] Berners-Lee, T.: "Weaving the Web". Harper Business, New York, 2000 ISBN: 006251587X

[2] J. Delgado, I. Gallego, R. García, R. Gil. "An ontology for Intellectual Property Rights: IPROnto", First International Semantic Web Conference, Sardinia, 2002

[3] Rust, G., C. Barlas.: "The MPEG-21 Rights Data Dictionary". IEEE Transactions on Multimedia, Vol. 7, No. 2, 2005
[4] Becket, D.: "RDF/XML Syntax Specification" W3C Recommendation. 2004 http://www.w3.org/TR/2004/RECrdf-syntax-grammar-20040210.

[5] Creative Commons, http://creativecommons.org

[6] Dublin Core Metadata Initiative, http://dublincore.org

[7] Iannella, R.: "Open Digital Rights Language (ODRL), Version 1.1" W3C Note, 2002 http://www.w3.org/TR/odrl

[8] Walle, R.v.d.: "The MPEG-21 Book". John Wiley \& Sons, Chichester, 2005

[9] HTML Tidy, http://tidy.sourceforge.net

[10] XQuery, http://www.w3.org/TR/xquery

[11] Dean, M. and Schreiber, G.: "OWL Web Ontology Language Reference". W3C Recommendation, 2004. http://www.w3.org/TR/owl-ref

[12] Pease, A.; Niles, I. and Li, J.: "The Suggested Upper Merged Ontology". AAAI Press, 2002. 1-57735-164-9

[13] <indecs>, http://www.indecs.org

[14] WIPO, http://www.wipo.int

[15] García, R.; Delgado, J. \& Gallego, I.: "RELOntos, REL Ontologies". ISO/IEC JTC1/SC29/WG11, Munich, Germany,No.M10703,2004

[16] García, R.; Delgado, J. \& Gallego, I.: "MPEG REL and RDD Ontologies API". ISO/IEC JTC1/SC29/WG11, Munich, Germany, No. M10702, 2004

[17] García, R.; Delgado, J. \& Gallego, I.: " Use of Semantic Tools for a Digital Rights Dictionary" (EC-Web 2004) Zaragoza (Spain). 2004.

[18] Wolf, Y., Karev, G. and Koonin, E.: "Scale-free networks in biology". Bioessays, 24, pp. 105-109, 2002

[19] Amaral, L.A.N., Scala, A., Barthélémy, M., Stanley, H.E.: "Classes of small-world networks". Proc. Natl. Acad. Sci, USA, 97, pp. 11149-11152, 2000

[20] Montoya J.M., Solé, R.V.: "Small World Patterns in Food Webs". J. Theoretical Biology, pp. 405-412, 2002

[21] Albert, R., Barabási, A.L.: "Statistical mechanics of complex networks". R. Modern Physics, 74, pp. 47-97, 2002

[22] Adamic, L.A.: "The Small World Web". Proceedings of ECDL'99, LNCS 1696, Springer-Verlag, 1999

[23] Barabási, A.L., Dezso, Z., Ravasz, E., Yook, S.H., Oltvai, Z.: Scale-free and hierarchical structures in complex networks. Proc. on Complex Networks, 2002

[24] Pool, I., Kochen, M.: Contacts and influence. Social Networks 1, pp. 1-48, 1978

[25] Milgram, S.: The small world problem. Psychology Today 2, pp. 60-67, 1967

[26] Solé, R.V., Ferrer, R., Montoya, J.M., Valverde, S.: Selection, tinkering and emergence in Complex Systems. Complexity, 8(1), pp. 20-33, 2002

[27] FOAF http://www.foaf-project.org

[28] DAML Library, http://www.daml.org/ontologies

[29] Pajek, http://vlado.fmf.uni-lj.si/pub/networks/pajek

[30] Kleinberg, J., Lawrence, S.: "The Structure of the Web". Science, Vol. 294, pp. 1849-1850, 2001

[31] Sigman, M., Cecchi, G.A.: "Global organization of the Wordnet lexicon". Proc. Natl. Acad. Sci, vol. 99, no. 3, 1742-1747, 2002

[32] Ferrer, R., Solé, R. V.: "The small world of human language". Proc The Royal Society 268, pp. 2261-2265, 2001 